\title{
MULTIPLE CHOICE QUESTIONS- PART II (CLASSIFICATION, ITEM PREPARATION, ANALYSIS AND BANKING)
}

Chaudhary $\mathrm{N}^{1}$, Bhatia BD ${ }^{2}$, Mahato $\mathrm{SK}^{1}$, Agrawal $\mathrm{KK}^{3}$

In the previous issue, we discussed single best response MCQs. In this section, we will discuss other forms of MCQs, item preparation, pre-validation process, item analysis and banking of MCQs.

\section{TYPES OF MCQS IN OBJECTIVE TESTING}

1. One best response type (most widely used and already discussed in previous issue)

2. K-type MCQs (next most widely used MCQ)

3. Matching type

4. Relationship analysis type

5. Case history type (Patient management problem)

6. Pictorial type

7. Multiple independent true-false selection type

1. Assistant Professor, Department of Pediatrics, Universal College of Medical Sciences \& Teaching Hospital, Bhairahawa, Nepal

2. Professor, Department of Pediatrics, Universal College of Medical Sciences \& Teaching Hospital, Bhairahawa, Nepal

3. Assistant Professor, Department of Medicine, Universal College of Medical Sciences \& Teaching Hospital, Bhairahawa, Nepal

For Correspondence

Dr. Nagendra Chaudhary

Assistant Professor,

Department of Paediatrics

Universal college of medical Sciences

Bhairahawa, Nepal

E-mail: enagendra@hotmail.com 


\section{K TYPE MULTIPLE CHOICE QUESTIONS (MCQS) Is it effective than standard single option MCQs?}

K-type multiple choice questions are also called combined response multiple choice questions or multiple-multiple choice questions.

\section{Anatomy of k-type MCQs}

K type MCQs have similarities with single response MCQs. It shares most of the advantages and disadvantages of a multiple choice questions (as discussed in previous communication). In items of the combined-response variety, one or more of the alternatives are correct answers; the remaining alternatives serve as distractors. The student is directed to identify the correct answer or answers by selecting one of a set of letters, each of which represent a combination of alternatives.

\section{The parts of K-type MCQs item are}

i) Stem

ii) 4 alternatives (One or more are correct)

iii) Incorrect responses are called as distractors which could be none or more

There cannot be all incorrect responses in these type of questions. At least one option should be correct response.

\section{Key pattern for correct answer is: " $e$ " in the given $M C Q$ example}

a) When 1,2, and 3 are correct

b) When 1 and 3 are correct

c) When 2 and 4 are correct

d) When 4 is correct

e) All of the above $(1,2,3,4)$ are correct

\section{Example:}

Edema is seen in:

1. Chronic liver disease

2. Nephrotic syndrome

3. Congestive cardiac failure

4. Kwashiorkor

\section{Purpose of K-type MCQs}

The k-type MCQs can be used to analyse, synthesize, and evaluate in order to select an answer. It can also be used to assess integration of knowledge and decision making.

\section{Guidelines for Constructing K-type Multiple-Choice Items:}

The guidelines for constructing item (stem/distractors/key) are similar to single response MCQs, as discussed in previous issue of the journal.

\section{Disadvantages:}

It has a disadvantage of providing clues that help students with even partial knowledge of subject to guess the correct combination of alternatives. In the example above, a student can identify combination " $e$ " as the correct response simply by knowing that alternatives 1 and 4 are both correct.

\section{MATCHING TYPE MCQs}

Directions: Each group of questions below consists of five lettered headings followed by a list of numbered words or phrases. For each numbered word or phrases, select ONE lettered heading that is most closely related to it and blacken the corresponding space in the answer sheet. Each lettered heading may be selected once, more than once, or not at all.

Example:

A. Increased metabolic activity

B. Hyper insulinism

C. Lack of storage of glycogen in liver

D. Storage of abnormal glycogen in liver

E. Decreased secretion by pituitary or adrenal gland

1. Adenoma of islets of Langerhans

2. Violent exercise

3. Hyperthyroidism

4. Simmond's disease

5. Von Gierke`s disease

6. Epidemic hepatitis

Key: A-2, 3 ; B-1; C-6; D-5; E-4

\section{RELATIONSHIPANALYSIS TYPE MCQs:}

This type tests reasoning and understanding of the basic principles, or the cause and effect relationship.

Direction: Each item below consists of two statements numbered (1) and (2) respectively. Please mark your response according to the instructions given below on the appropriate space in the answer sheet by blackening the space number.

A- If (1) is correct and (2) is correct and (1) and (2) are causally related.

B- If (1) is correct and (2) is correct and (1) and (2) are not causally related.

C- If(1) is correct and (2) is not correct

D- If (1) is not correct and (2) is correct

E- If ( 1 ) is not correct and (2) is not correct 
Example:

Item I:

(1) Staphylococcus aureus can cause food poisoning Because

(2) Staphylococcus aureus produces an exotoxin called enterotoxin

Key: A

\section{CASE HISTORY TYPE (PATIENT MANAGEMENT PROBLEM) MCQS}

Example:

A 5 year child presented with upper GI bleed. Past history of PRBC transfusion twice. No significant family history. On examination, he had anemia, massive splenomegaly with normal liver span. No evidence of ascites.

a) What is the most common likely diagnosis?

1. Wilson disease

2. Extra hepatic portal vein obstruction

3. Galactosemia

4. Tyrosinemia

b) Which is the drug used to treat acute upper GI bleed?

1. Somatostatin

2. Vitamin K

3. IV antibiotics

4. Lactulose

\section{PICTORIAL TYPE MCQs}

Diagrams, charts, photomicrographs, ECGs, may be used to elicit the knowledge of structure, function, the course of clinical situation and interpretation of data

Directions:

The question below consists of a numbered list of descriptive words accompanied with a sectional diagram of rectum and anal canal with certain parts indicated with letters. For each of the numbered word(s) select the matching lettered part on the diagram and blacken the circle under the corresponding alphabet on the answer sheet:

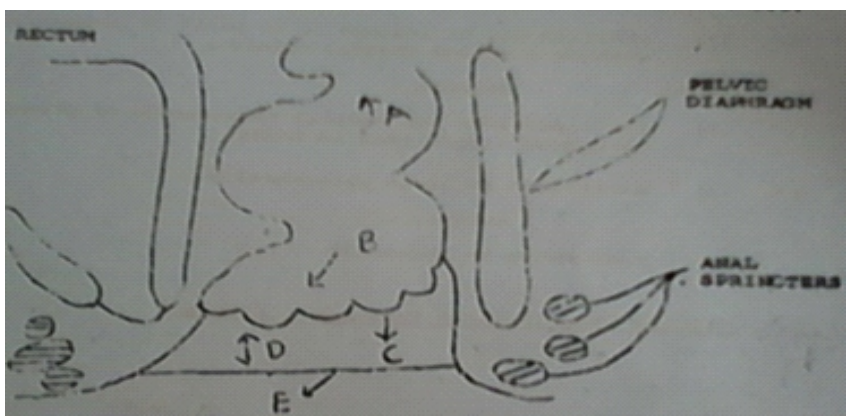

1. Pecten

2. Hilton's white line

3. Houstan`s valves

4. Pectinate line

5. Anal columns

\section{MULTIPLE INDEPENDENT TRUE-FALSE SELECTION TYPE MCQs}

Candidate has to answer separately to each of the four or five choices and is instructed to respond to each choice by "yes" or "No"/ "True" or "False".

Example:

Direction: To each of the alternatives for the item below you are to respond "Yes" or "No".

1. Greatly increased level of serum alkaline phosphatase is commonly found in:

a) Osteoporosis

b) Obstructive jaundice

c) Prostatic carcinoma with bony metastasis

d) Paget's disease of the bone

(Yes)

2. The consequences of breathing $5 \% \mathrm{CO}_{2}$ in air include

True False

a) Increased in mixed venous PCO2 ( ) ( $)$

b) Increase in PH of alveolar blood ( ) ( $)$

c) Increase in alveolar ventilation ( ) ( )

d) Decrease in cerebral blood flow ( ) ( )

e) Shift of the $\mathrm{O} 2$ dissociation curve to right

\section{ITEMS CAN BE PREPARED BY}

a) An individual: the quality of item is poor

b) Group of teachers: It is brainstorming and produces better quality of question.

c) a + b, i.e., itemsproduced by an individual teacher are usually incomplete and these can then be open for group discussion to remove ambiguity, providing good quality distractors and improve language so ultimately a good quality item is framed.

\section{Time for preparing an item}

While preparing for lesson or immediately after taking a lecture on a particular topic.

Best way to derive distractors: Derive distractors from 
student's incorrect responses and also from misconceptions evident in their answers. This is done while a teacher is evaluating answer sheet.

\section{Further improvement of an item by trying on group of} students followed by:

a) Discussion after they have been answered by students.

b) Advantages outweigh the disadvantages, for eg, ambiguities, errors in content, errors in construction, instances of contradictory teaching, triviality of content. The improved item can be used in subsequent tests.

Other way to improve an item is through an item analysis.

\section{ITEMANALYSIS}

- Identifies deficiencies in questions

- Questions are looked for importance of content. If not, revise questions to achieve desirable level of difficulty.

If weaker students answer correctly in greater number, then check the keying error, ambiguous in statement or misleading distractors.

\section{Pre validation process}

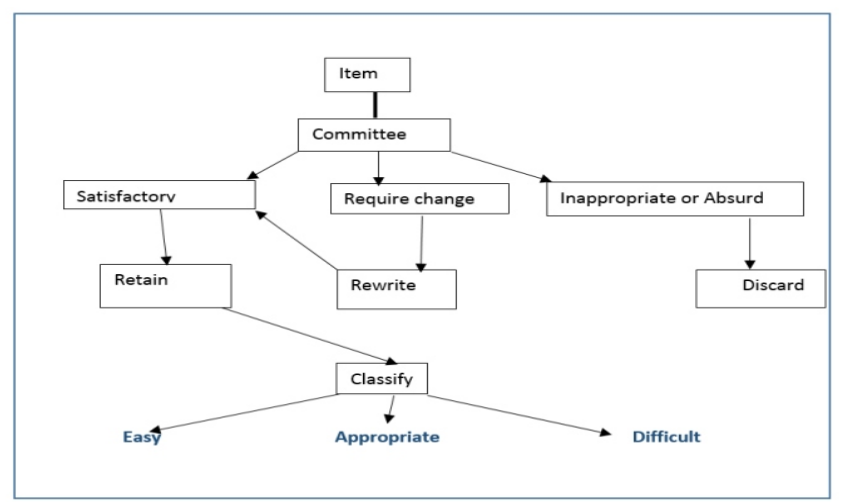

\section{INTERPRETATION OF ITEM ANALYSIS}

After an MCQ test has been administered, the students responses are evaluated. Item analysis includes:

1) Difficulty index or facility value

2) Discrimination index (the discrimination power of an item)

3) Distractor functionality or effectiveness

This will tell us whether MCQ,

-Is too easy or too difficult

-How well it discriminates between high and low scorers on test

-Whether all alternatives functional as intended
Questions with item difficulty of $50-60 \%$ are considered good, $30-70 \%$ are acceptable and those with indices above $70 \%$ or below $30 \%$ may require modification.

Questions with discrimination index of 0.35 and above are considered as excellent, 0.25-0.34 good, 0.2-0.25 acceptable and those below 0.2 require modification.

We should generally choose items at the $50 \%$ level of difficulty and those with highest discriminating power.

It is good practice to record item analysis data on a card and as well maintain in computer for banking and further improvement as well.

\section{Item analysis procedure}

We shall use 60 test papers to illustrate the steps

1. Arrange the 60 test papers results in rank order from the highest to lowest scores.

2. Select one third of the papers with highest scores (i.e., the first 20) and we call them as the upper group.

3. Select another one third papers with low scores (i.e., the last 20) and we call them as the lower group.

4. Set aside the middle thirdi.e., middle 20 values.

5. For each item, prepare a frequency table by counting the number of students in the upper group who selected each alterative; similarly count for lower group also.

Example: Item no

$\begin{array}{lcc}\text { Alternatives* } & \begin{array}{c}\text { No of responses in } \\ \text { Upper group }\end{array} & \begin{array}{c}\text { No. of responses in } \\ \text { Lower group }\end{array} \\ \text { A } & & \\ \text { B } & \\ \text { C } & \\ \text { D } & \\ \text { E } & & \end{array}$

No response

Total response $(\mathrm{T})$

*Encircle the correct answer for each item

6. Calculate the item difficulty, (P) by using the formula given below:

$$
\mathrm{P}=\frac{\mathrm{H}+\mathrm{L} \times 100}{\mathrm{~T}}
$$

Where, $\mathrm{H}=$ number of correct responses in the upper group $\mathrm{L}=$ number of correct responses in the lower group $\mathrm{T}=$ Total number of responses in both groups 
Note: $\mathrm{P}$ is usually expressed as a percentage. The lower the percentage figure, the more difficult is the item.

7. Calculate the index of discrimination (d) of item by using the formula,

$$
\mathrm{d}=\frac{2 \mathrm{X}(\mathrm{H}-\mathrm{L})}{\mathrm{T}}
$$

" $\mathrm{d}$ " is reported as a decimal fraction. Maximum positive discrimination power is indicated by 1.00 and minimum is 1.00 (negative discriminant).

\section{Interpretation of Analysis data}

1. Difficulty index:

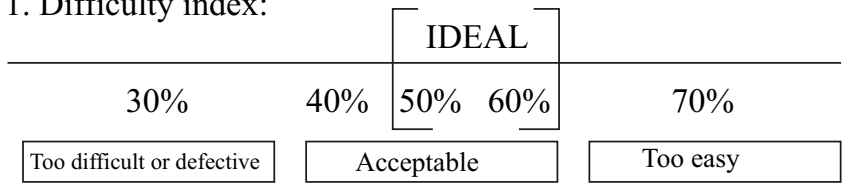

1. Discrimination index:

\begin{tabular}{cccc} 
Revise & Acceptable & Good & Excellent \\
\hline 0.15 & 0.20 & 0.25 & 0.35
\end{tabular}

\section{HOW TO ORGANIZE AMCQ BANK?}

- Creation of good questions is not an easy task

- To construct a fair MCQ paper is still more difficult

The practice of creating new questions each time and discarding them after examination is over, is WASTE of effort and INEFFICIENT use of an academician`s time.

Banks of questions increase efficiency in the use of MCQs and improvement of their quality.

\section{HOW CAN QUESTIONS BE STORED?}

\section{A) Several classifications could be used:}

1. Subdivision of subject: Eg, in Pediatrics- Neonatology, Nutrition, Growth and development, haematology etc. In Anatomy: Gross anatomy, histology, embryology etc.

2. Body systems: Cardiovascular, Respiratory, Central nervous systems, etc.

3. Level of knowledge to be tested: Recall, Application, Understanding

4. Type of item: Single response, multiple response, true/false, reason/assertion, matching etc.
B) Assign a number to each question and code to identify nature of question

Fig: $\frac{\mathbf{1}}{\text { Type of question }} \frac{\mathbf{2}}{\text { Body }} \frac{\mathbf{3}}{\text { Assigned in series }}$

Computerized banking (Database) or store cards can be used. This task should not be taken lightly. Cooperation of all but commitment of one is required in proper storage of question banks.

\section{REFERENCES:}

1. Cox KR, Ewan CE. The medical Teacher. London, Churchill Livingston, 1982. Chapter 37. Writing multiple choice questions. Bandarnaike RC, Cox KR. Chapter 38. How to construct a fair multiple Choice question paper? Cox KR. Chapter 39. What can teachers and students get out of multiple choice questions. Cox KR

2. Cox KR. How didyou guess? Med. J. August 1976; 1: 884-86.

3. Ebel RL. Essentials of educational management: Englewood Cliffs; New Jersey, prentice-Hall Inc. 1972

4. Anderson J. For Multiple choice questions. Medical teacher 1979;1:37-42.

http://dx.doi.org/10.3109/01421597909014340

http://dx.doi.org/10.3109/01421597909010580

PMid: 24483175

5. Picketting G. Against multiple choice Questions. Medical teacher 1979; 1: 84-86.

http://dx.doi.org/10.3109/01421597909019397

PMid:24479932

6. Hubbart JP, Clemans WV. Multiple Choice Examinations in Medicine. Philadelphia: Lea and Febiqer; 1961.

7. Hubbart JP. Measuring medical education. Philadelphia: Lea and Febiqer; 1981.

\section{Answers to the previous issue MCQs}

1. It includes double negatives which should be avoided. Item could be as: Which of the following is a loop diuretics?

2. It gives additional clues like pneumothorax and hydrothorax.Item could be as: An intercostal tube may be placed in a patient with flail chest to...

3. It includes repetition of similar words in each distractors. Stem could be written as: Protein requirement of Indian 
reference man in $\mathbf{g m} / \mathbf{k g} / \mathbf{d a y}$ is:
a) 1
b) 2
c) 3
d) 4

4. It includes repetition of the word congenital in Distractors
(a) and
(b) which should be avoided.

5. The stem is too long.

6. It includes EXCEPT and none of the above, which should always be avoided.

7. The stem gives a clue (an), so the students can guess as an antidepressant.

8. The distractors are not of same length in this item.

9. The numerical values in distractors should be similar, eg., $1.3,2.3,3.3,4.3$

10. In this item posterior pituitary and pars nervosa are similar, which should not be used.

SUBSCRIPTION CHARGES

\begin{tabular}{|c|c|c|}
\multicolumn{1}{c}{} & \multicolumn{1}{r}{$\begin{array}{r}\text { INSTITUTION } \\
\text { Single copy/Annual }\end{array}$} & $\begin{array}{c}\text { INDIVIDUAL } \\
\text { Single copy/Annual }\end{array}$ \\
\hline NEPAL & NRS. 300/1000 & NRS. 200/700 \\
\hline SAARC COUNTRIES & IRS. 300/1000 & IRS.200/700 \\
\hline OTHER COUNTRIES & US\$ 40/100 & US\$ 20/75 \\
\hline
\end{tabular}

ADVERTISEMENT CHARGES

\begin{tabular}{|c|c|c|}
\hline S.NO & PAGE & RATE (NC) \\
\hline 1. & FULL BACK COVER & 15000 \\
\hline 2. & FULL INSIDE OF FRONT COVER & 12000 \\
\hline 3. & FULL INSIDE & 10000 \\
\hline 4. & HALF SIDE & 8000 \\
\hline 5. & QUARTER & 4000 \\
\hline
\end{tabular}

